\title{
Review
}

\section{Personalized Medicine for the Management of RDS in Preterm Neonates}

\author{
Daniele De Lucaa, b Chiara Autilioc Lucilla Pezzad Shivani Shankar-Aguilera ${ }^{\mathrm{a}}$ \\ David G. Tingay ${ }^{\mathrm{e}} \mathrm{f}, \mathrm{g}$ Virgilio P. Carnielli ${ }^{\text {h }}$ \\ aDivision of Paediatrics and Neonatal Critical Care, "A. Béclère" Medical Centre, Paris Saclay University Hospitals, \\ APHP, Paris, France; bPhysiopathology and Therapeutic Innovation Unit-INSERM U999, Paris Saclay University, \\ Paris, France; 'Department of Biochemistry and Molecular Biology and Research Institute "Hospital 12 de Octubre,"

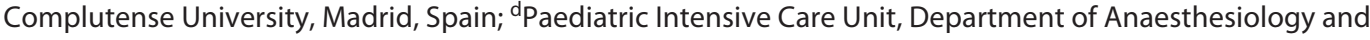 \\ Critical Care, University Hospital "A. Gemelli"- IRCCS, Rome, Italy; eNeonatal Research, Murdoch Children's Research \\ Institute, Melbourne, VIC, Australia; 'Department of Neonatology, Royal Children's Hospital, Melbourne, VIC, \\ Australia; ${ }^{9}$ Department of Paediatrics, University of Melbourne, Melbourne, VIC, Australia; ${ }^{\text {h}}$ Division of Neonatology, \\ "G. Salesi" Women and Children Hospital, Polytechnical University of Marche, Ancona, Italy
}

\section{Keywords}

Prematurity $\cdot$ Infant $\cdot$ Continuous positive airway pressure

\begin{abstract}
Continuous positive airway pressure and surfactant represent the first- and second-line treatment for respiratory distress syndrome in preterm neonates, as European and American guidelines, since 2013 and 2014, respectively, started to recommend surfactant replacement only when continuous positive airway pressure fails. These recommendations, however, are not personalized to the individual physiopathology. Simple clinical algorithms may have improved the diffusion of neonatal care, but complex medical issues can hardly be addressed with simple solutions. The treatment of respiratory distress syndrome is a complex matter and can be only optimized with personalization. We performed a review of tools to individualize the management of respiratory distress syndrome based on physiopathology and actual pa-
\end{abstract}

tients' need, according to precision medicine principles. Advanced oxygenation metrics, lung ultrasound, electrical impedance tomography, and both quantitative and qualitative surfactant assays were examined. When these techniques were investigated with diagnostic accuracy studies, reliability measures have been meta-analysed. Amongst all these tools, quantitative lung ultrasound seems the more developed for the widespread use and has a higher diagnostic accuracy (meta-analytical AUC $=0.952$ [95\% Cl: 0.951-0.953]). Surfactant adsorption (AUC $=0.840$ [95\% Cl: 0.824-0.856]) and stable microbubble test (AUC $=0.800$ [95\% Cl: $0.788-$ $0.812]$ ) also have good reliability, but need further industrial development. We advocate for a more accurate characterization and a personalized approach of respiratory distress syndrome. With the above-described currently available tools, it should be possible to personalize the treatment of respiratory distress syndrome according to physiopathology.

(c) 2021 S. Karger AG, Basel karger@karger.com

(c) 2021 S. Karger AG, Basel

www.karger.com/neo

Karger ${ }^{\prime}=$
Daniele De Luca

Service de Pédiatrie et Réanimation Néonatale

Hôpital "A. Béclère" - GHU Paris Saclay, APHP

157 rue de la Porte de Trivaux, Clamart, FR-92140 Paris-IDF (France)

dm.deluca@icloud.com 


\section{Introduction}

\section{Background}

Continuous positive airway pressure (CPAP) is internationally recognized as the first-line therapy for respiratory distress syndrome (RDS) in preterm neonates: early CPAP significantly reduces mortality and bronchopulmonary dysplasia [1]. This has resulted in a change in the recommendations for surfactant replacement from the long-held "prophylaxis" approach for RDS: European and American guidelines, since 2013 and 2014, respectively, recommended surfactant replacement only when CPAP fails $[2,3]$.

This creates a clinical dilemma, as surfactant is more effective in decreasing mortality and/or bronchopulmonary dysplasia when given within the first $2-3 \mathrm{~h}$ of life [4]. One argument is that clinicians should give surfactant as early as possible in those neonates who fail CPAP. The administration after the window of maximal surfactant benefit should be avoided but still occurs in approximately $30 \%$ of cases [5]. Alternatively, surfactant, a valuable medical resource not without administration risks, should only be used in those neonates whose respiratory failure cannot be managed with CPAP alone. Thus, RDS is quite variable in its clinical course and seems likely to benefit from a personalized medicine approach. Almost 50 and 40 years from the first clinical use of CPAP [6] and surfactant [7], respectively, the essential question as to "who are the CPAP-treated preterm neonates with RDS that will actually benefit from surfactant?" remains unanswered. Meanwhile, the introduction of prenatal steroid prophylaxis has also significantly changed the clinical severity of RDS and improved clinical outcomes [8], while several technologies have become available to characterize the physiopathology of respiratory failure in preterm neonates.

Thus, the time has come for surfactant therapy to enter into the era of personalized medicine to improve its efficacy, as the individual patient needs and risk/benefit ratio may significantly vary. Several tools may be used to tailor the treatment to the individual physiopathology during the evolution of RDS: we offer here a comprehensive review of these tools trying to answer the aforementioned question in the context of modern optimal perinatal care.

\section{What Is CPAP Failure?}

It is pivotal to understand how we can define CPAP failure and, consequently, identify an infant that might need surfactant replacement. For the sake of this review, we will refer to CPAP failure caused by worsening RDS, that is, by increased severity of respiratory failure needing surfactant as a second-line treatment. Thus, we will not consider CPAP failure due to other reasons, such as severe apnoea or haemodynamic impairment, which are characterized by a different physiopathology.

RDS severity can be described in several ways, and the commonest one is a combination of (1) inspired oxygen fraction $\left(\mathrm{FiO}_{2}\right)$ needed to maintain peripheral saturation within a given range and (2) the clinical judgement of work of breathing (WOB) [3]. WOB is correlated with diaphragm electrical activity in infants [9], but its measurement is not widely available. Both $\mathrm{FiO}_{2}$ and $\mathrm{WOB}$ are influenced by the level of CPAP. The 2019 edition of the European guidelines suggests to apply at least $6 \mathrm{cmH}_{2} \mathrm{O}$ of CPAP [10], while the American Academy of Paediatrics and the World Health Organization did not provide details in their statements $[2,11]$. The American Association of Respiratory Care clinical guidelines acknowledge that $\mathrm{CPAP}$ level may vary between 4 and $10 \mathrm{cmH}_{2} \mathrm{O}$ and should be titrated according to patients' condition [12]. Trials are ongoing to clarify what is the ideal amount of pressure to apply [13], but, to date, the optimal pressure level is unknown and is likely to be different between patients.

Thus, there is a trend to use $\mathrm{FiO}_{2}$ thresholds alone to indicate the need for surfactant administration in RDS [3]. However, using $\mathrm{FiO}_{2}$ alone cannot accurately describe the severity of respiratory failure and entails an important loss of information if we do not consider at least target saturation and/or WOB. Moreover, the $0.30-\mathrm{FiO}_{2}$ threshold suggested by European guidelines [10] is based on a single-centre, retrospective study [14]. Other studies with similar design have proposed different thresholds with comparable accuracy [15]. Unsurprisingly, the American Academy of Paediatrics does not recommend any particular $\mathrm{FiO}_{2}$ thresholds to give surfactant [2]. Moreover, $\mathrm{FiO}_{2}$ critical values might be attained well after the 3 -h optimal time-window for surfactant administration, thus reducing the efficacy of surfactant replacement [4].

Furthermore, $\mathrm{FiO}_{2}$ does not accurately describe the "oxygenation," as the latter is a more complex concept influenced by other factors, such as CPAP level (and pressure leaks), arterial oxygen content, total and foetal haemoglobin levels, temperature, degree of lung development, peripheral perfusion, and cardiac output. Some of these factors may be somewhat homogeneously controlled by haemodynamic management and incubators. Others are variable and unpredictable, such as foetal haemoglobin [16], or constant but important, such as pressure leaks when the mouth is open [17].

Let us imagine two neonates of 26-week gestation with RDS (Fig. 1). According to suggested $\mathrm{FiO}_{2}$ levels [10], 


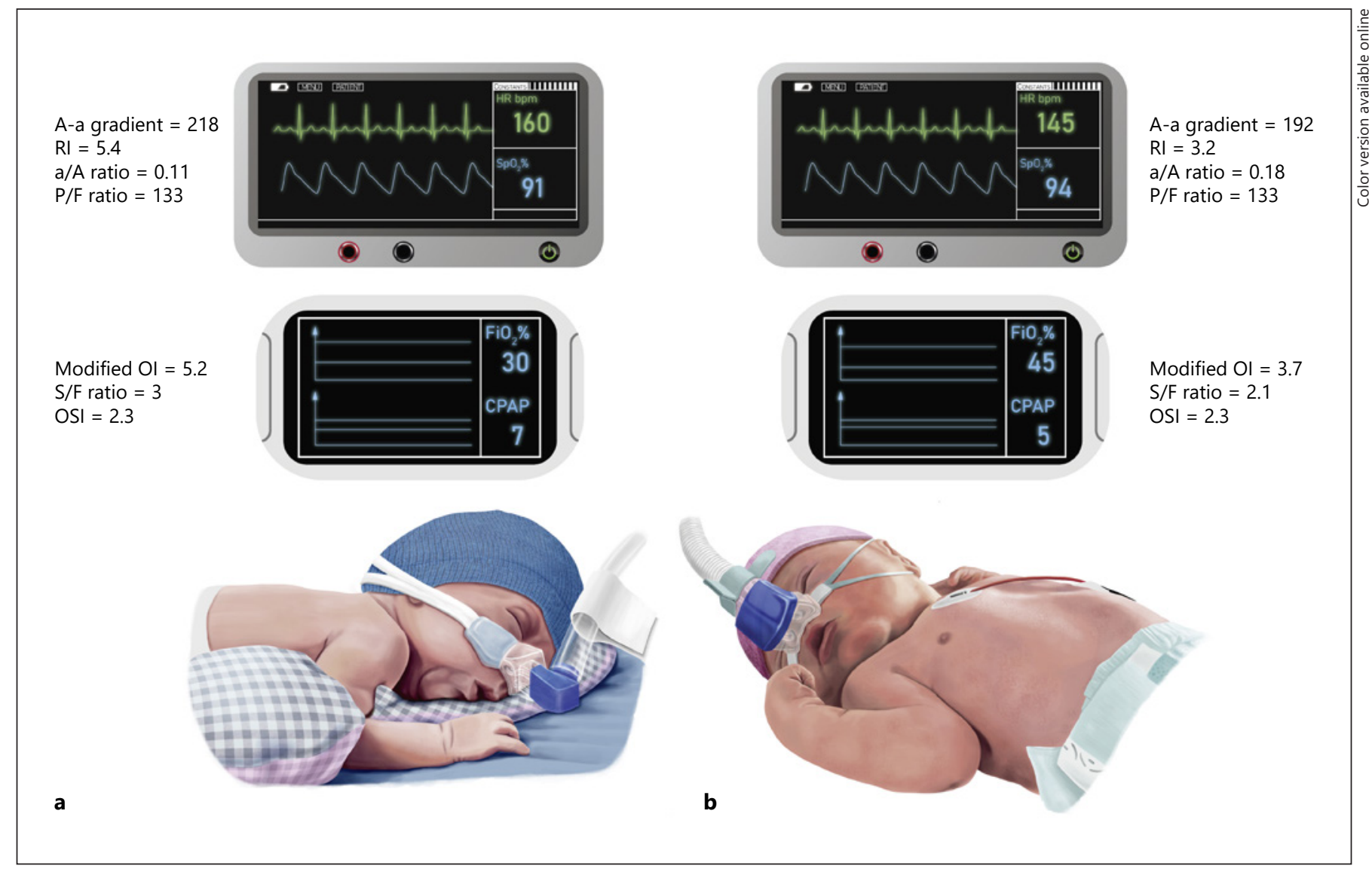

Fig. 1. Clinical cases illustrating the difficulty to evaluate the severity of RDS based on $\mathrm{FiO}_{2}$ alone. Tommy, a baby receiving $7 \mathrm{cmH}_{2} \mathrm{O}$ of CPAP with an $\mathrm{FiO}_{2}$ of 0.30 (a); Laura, a baby under $5 \mathrm{cmH}_{2} \mathrm{O}$ of CPAP and 0.45 of $\mathrm{FiO}_{2}$ (b). Tommy and Laura have the same gestational age (26 weeks), both have the mouth partially open and are treated with the same type of nasal mask-delivered CPAP. Advanced oxygenation metrics have been calculated considering a $\mathrm{PaO}_{2}$ of 40 and $60 \mathrm{~mm} \mathrm{Hg}$ and $\mathrm{PaCO}_{2}$ of 50 and $55 \mathrm{~mm} \mathrm{Hg}$, for Tommy and Laura, respectively. Foetal haemoglobin content and pressure leaks have not been considered (more details on the cal-

Laura should receive surfactant, while Tommy does not, but who is the sickest? This is impossible to say without considering some of the aforementioned variables: the CPAP level, the leaks, and the oxygen tension, at the very least. Some of these variables are considered in advanced oxygenation metrics that may be used to accurately answer this question and will be described further in this review. Consistently, severe respiratory failure in adults and children $[18,19]$ is not evaluated by using only $\mathrm{FiO}_{2}$, as it does not represent a criterion in the definition of neonatal, paediatric, or adult acute respiratory distress syndrome (ARDS) $[18,20,21]$. It seems clear that CPAP

Personalization of RDS Management Is Possible culations are available in the text). It is misleading to say that Lau$\mathrm{ra}$ is sicker than Tommy only because of her higher $\mathrm{FiO}_{2}$. Tommy's oxygenation is worse than Laura's according to A-a gradient, RI, a/A ratio, modified OI, and S/F ratio; conversely, $\mathrm{P} / \mathrm{F}$ ratio and OSI indicate an identical oxygenation impairment. RDS, respiratory distress syndrome; CPAP, continuous positive airway pressure; A-a gradient, alveolar-arterial gradient; a/A, arterial/alveolar ratio; $\mathrm{FiO}_{2}$, inspired oxygen fraction; modified OI, modified oxygenation index; $\mathrm{P} / \mathrm{F}, \mathrm{PaO}_{2} / \mathrm{FiO}_{2}$ ratio; $\mathrm{S} / \mathrm{F}$, peripheral saturation $/ \mathrm{FiO}_{2}$ ratio; OSI, oxygen saturation ratio.

and surfactant replacement are presently "crude" therapies: simplification of treatment algorithms may be beneficial when initiating new therapies to clinical practice, but then complex medical problems cannot be solved with easy solutions.

\section{Methods to Analyse Candidate Tools for Personalized Medicine}

Protocol

Prior to commencing the project, a protocol was agreed to determine the database to be searched, search details, eligibility cri- 
teria, data collection and analysis, and methods for dispute resolution in case of disagreement. We a priori chose to focus on the following tools:

- Advanced oxygenation metrics

- Functional lung imaging

- Surfactant biological tests

\section{Search Details}

For each of these, a literature search has been performed on PubMed (on October 7, 2020), combining them with CPAP failure and surfactant, as words or MeSH terms, without year or language restrictions but limited to the first month of life. We also handsearched references cited in the studies identified through the initial search, review articles on the subject, and the authors' personal archives.

\section{Eligibility Criteria}

We searched for diagnostic accuracy studies aiming to predict CPAP failure due to surfactant need in the context of modern perinatal care (i.e., with adequate perinatal monitoring, prenatal steroids, and early CPAP delivered by short binasal prongs or masks). We excluded "grey" literature, unpublished, or nonpeer reviewed reports. Details of selected studies were included in a customized database, removing duplicates, which were used for the following analyses.

\section{Data Items and Summary Measures}

We collected sensitivity, specificity, area under the curves (AUC), and population size of each study into a customized data extraction sheet. Interpretation discrepancies, if any, were resolved through discussion between the authors. When more than one study was available on a given tool, their diagnostic accuracy measures were pooled with the DerSimonian-Laird random-effects model and the inverse variance method. Such an approach is more conservative than the fixed-effects model since it recognizes the presence of heterogeneity because studies estimate different underlying effect sizes. Consistency between the studies was estimated with the $I^{2}$ value (i.e., variation in sensitivity and specificity due to heterogeneity) and evaluated by $\chi^{2}$ test; $I^{2}$ values $>50 \%$ were considered indicative of significant heterogeneity. Meta-regressions were performed adjusting for gestational age and male sex as they may be confounders [22]. These analyses were done using raw data extracted from articles or requested from authors, when necessary. We inserted one covariate in each model in order to reduce false-positive results [23]. AUC and relative diagnostic odds ratios (RDOR) were reported with 95\% confidence interval (95\% CI). $p$ values $<0.05$ were considered to be statistically significant. Analyses were performed with Meta-Disc 1.4 and Open-MetaAnalyst $10.1[24]$.

\section{Advanced Oxygenation Metrics}

Complex oxygenation metrics allow consideration of factors influencing oxygenation other than $\mathrm{FiO}_{2}$. Advanced oxygenation metrics include the alveolar-arterial gradient \{calculated as $\mathrm{PA}-\mathrm{PaO}_{2}$, where $\mathrm{PA}$ indicates alveolar partial pressure (estimated, at the sea level, as
$\left.\left.\left(\mathrm{FiO}_{2} \times[760-47]\right)-\left[\mathrm{PaCO}_{2} / 0.8\right]\right)\right\}$ and the respiratory index (calculated as the $\mathrm{A}$-a gradient divided by $\mathrm{PaO}_{2}$ ). While these metrics are quite cumbersome to calculate and seldom used, the arterial/alveolar ( $\mathrm{a} / \mathrm{A}$ ) oxygen ratio and the $\mathrm{PaO}_{2} / \mathrm{FiO}_{2}(\mathrm{P} / \mathrm{F})$ ratio have been more diffused. The $\mathrm{a} / \mathrm{A}$ ratio has been used for many years using various thresholds to indicate surfactant replacement [25], and the ARDS definition in adults is still based on P/F thresholds [18]. In neonatology and paediatrics, the oxygenation index (OI) is also commonly used, as it takes the mean airway pressure into account $(\mathrm{OI}=$ mean airway pressure $\times \mathrm{FiO}_{2} \times 100 / \mathrm{PaO}_{2}$ ). Each of these metrics has peculiar characteristics and describes oxygenation from a different perspective; none of them can be considered a gold standard although they surely characterize the oxygenation impairment better than the mere $\mathrm{FiO}_{2}$ [26]. All these metrics include arterial $\mathrm{PaO}_{2}$ measured by blood gas analysis on samples obtained by indwelling arterial lines or by well-performed arterial puncture. This is not always technically possible and may be ethically questionable in neonates with mild respiratory failure not needing invasive procedures.

Nonetheless, these metrics may be modified to include less-invasive estimation of factors influencing oxygenation. For example, OI can be modified using CPAP set at the ventilator and transcutaneously measured $\mathrm{PaO}_{2}$ to estimate the mean airway pressure and arterial $\mathrm{PaO}_{2}$, respectively [21]. Transcutaneous devices are widely available for the non-invasive estimation of $\mathrm{PaO}_{2}$, and the American Association of Respiratory Care recognizes their accuracy, if adequately calibrated and used according to defined criteria [27]. As the measurement does not need to be continuous, leaks can be transiently reduced during the measurements, and the application of a transcutaneous probe at $43 / 44^{\circ} \mathrm{C}$ for $10-15$ min usually avoids any vasodilation-induced rash or skin injury. The paediatric ARDS definition also suggests to use the peripheral saturation $/ \mathrm{FiO}_{2}(\mathrm{~S} / \mathrm{F})$ ratio to evaluate patients' oxygenation [20]. In populations without foetal haemoglobin (adults and children), the S/F has an accuracy similar to the $\mathrm{P} / \mathrm{F}$ ratio $[28,29]$. The $\mathrm{S} / \mathrm{F}$ ratio without foetal haemoglobin correction has been recently used to define need for surfactant replacement in neonates [30]. Similarly, OI can be estimated without $\mathrm{PaO}_{2}$ measurements, by using an oxygen saturation index (OSI) replacing $\mathrm{PaO}_{2}$ with peripheral saturation. OSI correlates well with OI and A-a gradient, but the influence of foetal haemoglobin on these indexes is unknown $[31,32]$. An estimation of foetal haemoglobin might be included to correct saturation values and further personalize the evaluation. 
OI is already commonly used to define neonatal or paediatric ARDS and decide about some interventions (such as nitric oxide or extracorporeal life support), while optimal peripheral saturation in preterm neonates has been extensively investigated: therefore, modified OI, S/F ratio, or OSI are easy to diffuse in neonatal critical care. Even though these modified indexes do not consider all factors influencing oxygenation or only estimate some of them, they describe oxygenation impairment more in detail than the mere $\mathrm{FiO}_{2}$. Oxygenation metrics may variously identify the sickest patient in the example provided in Figure 1: thus, we need studies to compare their ability to predict CPAP failure and notably the reliability of modified OI, S/F, and OSI and the influence of foetal haemoglobin to this end. Given the simplicity and availability of these tools and their solid physiopathology background, these studies are urgently warranted.

\section{Functional Lung Imaging: Lung Ultrasound}

Lung ultrasound can be used both for descriptive and functional purposes [33]. Functional lung ultrasound has been used as a point-of-care imaging tool to monitor acute respiratory failure in adults since decades, but it has only recently been introduced in neonatology [33]. The technique has been used to predict CPAP failure and surfactant need through a simple and qualitative evaluation of lung ultrasound findings [3436] or the calculation of a quantitative lung ultrasound score (LUS) modified from adult experience and specifically validated in neonates [37-42]. Lung ultrasound has always been performed in the first hours of life. Both methods resulted in good diagnostic accuracy on earlier meta-analysis [43]; however, the quantitative score is a more objective, refined, and rigorous way to evaluate the respiratory failure and is already widely used in adult critical care [44]. Figure 2 represents the summary receiver operator characteristics curve of diagnostic accuracy studies about quantitative LUS and CPAP failure: the meta-analytical AUC was 0.952 (95\% CI: 0.951-0.953), with an LUS cutoff value between 6 and 8 . This result is independent of gestational age (RDOR: 0.95 [95\% CI: 0.32-2.82], $p=0.890$ ) and sex (RDOR: 0.99 [95\% CI: 0.87-1.14], $p=0.906$ ). Interestingly, LUS-guided surfactant administration (also called Echography-guided Surfactant THERapy, ESTHER) has been already investigated in a quality improvement project and a randomized controlled trial

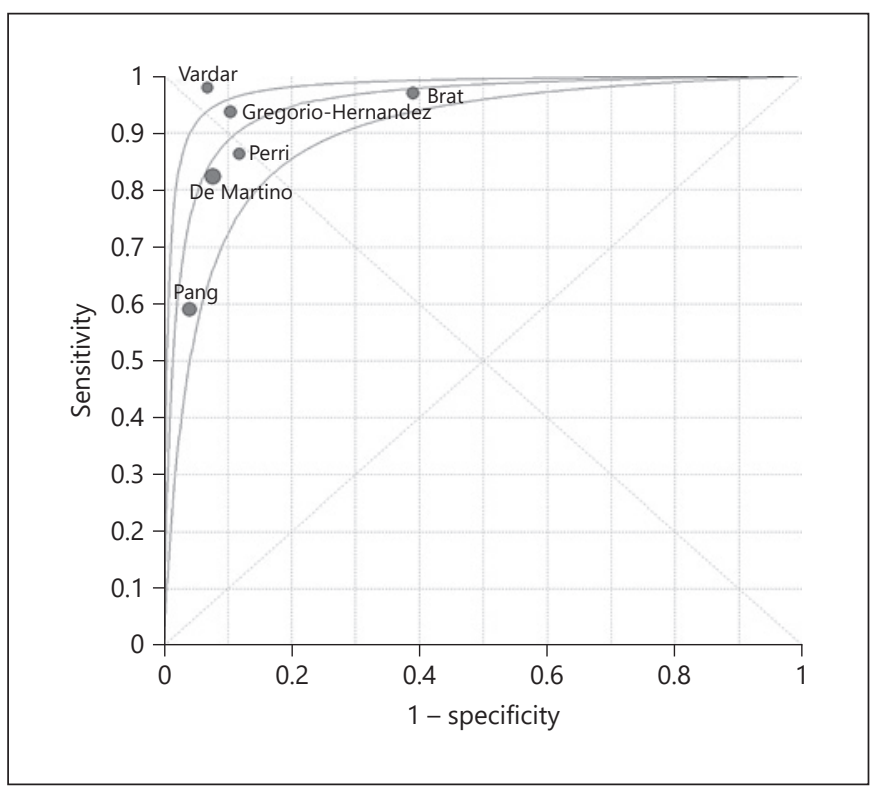

Fig. 2. Meta-analytical summary ROC curve of diagnostic accuracy studies [37-42] on LUS to predict CPAP failure. ROC analysis gave a pooled sensitivity and specificity of 84 (95\% CI: 77-89\%, $I^{2}$ : $78 \%)$ and 93 (95\% CI: 88-95\%, $\left.I^{2}: 84 \%\right)$, respectively $(p<0.0001$ for both), with an LUS cutoff value between 6 and 8. Data were obtained from the original article or asking directly to authors, and the curve has been fitted using Moses (weighted regression, inverse variance) model. Circles indicate the single studies, and the circle diameter is proportional to their weight; the first author name is indicated for each study. Grey curves indicate the summary ROC curve and its 95\% CI. LUC, lung ultrasound score; CPAP, continuous positive airway pressure.

providing consistent results $[5,30]$. ESTHER improves the timeliness of surfactant replacement and significantly reduces the number of patients needing it after $3 \mathrm{~h}$ of life; LUS-guided surfactant administration also improves oxygenation after surfactant dosing and reduces oxygen exposure early in life with possible secondary benefits, such as shorter invasive ventilation [5, 30]. Conversely, a large retrospective analysis on approximately 1,000 neonates demonstrated that ESTHER did not change the proportion of preterm neonates receiving surfactant or produce any significant cost benefit [45]. LUS also correlates with the lung inflammation and extravascular lung water in infants [46, 47]. Therefore, LUS is not specific to loss of lung aeration due to the magnitude of primary surfactant deficiency but can be useful also in cases of neonatal ARDS. This is consistent with the wide experience on lung ultrasound in adult critical care, as LUS is commonly used 
to measure lung aeration in patients with ARDS, that is, with a more complex surfactant and epithelial injury [48].

Until recently, chest radiography has been the only routinely available bedside neonatal lung imaging technique. Despite providing diagnostic information, chest radiography is not a functional lung imaging; moreover, interpretation has a high inter-operator variability and is inaccurate in respect to the clinical evolution [49]. A recent study demonstrated that a standardized radiological score for RDS was very variable in neonates failing CPAP and needing surfactant replacement [50]. To the best of our knowledge, only one formal diagnostic accuracy study compared chest X-rays and lung ultrasound to predict CPAP failure in the context of modern perinatal care: the authors found that radiography (AUC: 0.8 [95\% CI: $0.792-0.808]$ ) has significantly lower diagnostic accuracy than LUS [39].

Lung ultrasound also has additional interesting characteristics. It may reduce radiation exposure [51], and basic lung ultrasound semiology is successfully recognized with any type of probe and with high agreement between physicians of different expertise $[52,53]$ while the learning curve is steep also in developing countries [54]. Consistently, lung ultrasound has been recently listed amongst the point-of-care ultrasound to be used in paediatric and neonatal critical care whose evidence-based guidelines have been recently released [55].

\section{Functional Lung Imaging: EIT}

Electrical impedance tomography (EIT) is a method of dynamically imaging regional ventilation and lung function that holds promise in individualizing respiratory therapies. EIT is a validated research tool, with a recent international consensus statement to guide usage [56]. EIT uses the differential properties of air and liquids in tissues on electrical current: measurements of these properties are made via a small non-adhesive electrode belt placed around the infants' chest. EIT systems reconstruct the patterns of electrical activity to create an image of the relative amounts of ventilation, aeration, gas flow, or perfusion with the cross-sectional area of the chest imaged, and how these change over time [56].

EIT has been used to describe the effect of surfactant replacement, demonstrating improved uniformity of ventilation within the lungs during and after the treatment [57] and the relationship between surfactant and different respiratory support strategies $[58,59]$. Recently,
EIT was able to demonstrate differences in end-expiratory lung volume at different CPAP levels in preterm infants with RDS, with a precision that would not be possible based on oxygenation [60].

As real-time display clinical systems become available, EIT is used in adult and paediatric intensive care units, and its potential to personalize RDS management becomes attractive. In fact, similar to lung ultrasound, EIT might allow for more rapid identification of infants not responding to CPAP. Nonetheless, so far EIT has not been investigated in diagnostic accuracy studies specifically dedicated to the prediction of CPAP failure. Thus, compared to lung ultrasound, EIT represents an interesting but less-advanced tool on the clinical development pathway.

\section{Surfactant Biological Tests}

There are many biological tests able to measure the amount or the quality (function) of surfactant. If accurate, quick, and easy to use, a test of this kind could become a point-of-care technique at the bedside. Such a test would be similar to the point-of-care assays for C-reactive protein or procalcitonin to guide antibiotic therapy. Several tests measuring both the amount and the function of endogenous surfactant have been studied to predict RDS occurrence. For this review, we will only refer to those that have been formally investigated in diagnostic accuracy studies to predict $\mathrm{CPAP}$ failure due to surfactant need. A new point-of-care spectroscopic method to measure lecithin-sphingomyelin ratio in gastric aspirates has been recently proposed, but this has not been investigated to predict CPAP failure in diagnostic accuracy studies [61].

\section{Quantitative Tests}

Lamellar body count (LBC) is probably the easiest rapid biological surfactant test available. Lamellar bodies are storage particles consisting of packed surfactant phospholipids and proteins, released from type II alveolocytes [62]. Once in the alveoli, lamellar bodies open up with the compression/expansion cycles and create the surfactant film adsorbed at the gas/liquid interface [63]. Thus, $\mathrm{LBC}$ is an estimation of the available endogenous surfactant. LBC has been performed in amniotic fluid, gastric aspirate, and non-bronchoscopic bronchoalveolar lavage (BAL) samples [64] and predicts the occurrence of RDS with better accuracy than lecithin-sphingomyelin ratio [65]. LBC may actually be done at the bed- 
side, since lamellar bodies have the same diameter as platelets and can be counted by automated cell counters used in any hospital, without any dilution or sample preparation [64]. Unfortunately, LBC in gastric aspirate samples obtained in the first hours of life is not accurate enough to reliably predict CPAP failure due to surfactant need (AUC: 0.703 , 95\% CI: 0.696-0.710). It is also unsuitable, as the assay could not be performed in approximately $35 \%$ of samples due to viscosity or blood contamination [66]. LBC has also been investigated in a randomized clinical trial to guide surfactant replacement: it was unfeasible in $23 \%$ of patients for these technical problems and failed to improve the primary outcome (i.e., need for invasive ventilation or mortality) [67]. High viscosity or sample contamination is also likely to be observed in amniotic fluid samples collected at delivery, and especially those collected vaginally have shown reduced reliability [64].

This may be explained as LBC only estimates the surfactant amount available at birth: this is physiopathologically linked to the insurgence of RDS, rather than to its clinical evolution (i.e., need for surfactant or invasive ventilation). Consistently, phosphatidylcholine concentrations in foetal lung fluid do not correlate with RDS clinical severity in preterm lambs [68]. The same problem can affect other quantitative surfactant assays performed at the birth, since, beyond the originally available endogenous surfactant pool, the natural course of RDS is influenced by the CPAP levels, which recruit the alveoli and influence surfactant production [69]. The evolution of RDS is also influenced by other factors, such as the prenatal steroids dose [70], degree of alveolarization, amount of lung tissue inflammation, and extravascular water [71]. Some of these factors impact lung aeration and correlate with LUS $[46,47]$ : this makes LUS even more interesting, since LUS may give a more comprehensive picture of the lung aeration, taking all these factors into consideration.

\section{Qualitative Tests}

A qualitative might be more informative than a quantitative surfactant test, since it describes surfactant function rather than its amount. In fact, surfactant function, like lung aeration, is influenced not only by the available surfactant pool but also by its dilution in the extravascular lung water [72]. There are several techniques to study surfactant function, but the more interesting for our purposes are the surfactant adsorption test (SAT) and the stable microbubble test (SMT).

Personalization of RDS Management Is Possible
SAT has been successfully used for research purposes in BAL fluids obtained from animals and human neonates [73]. SAT consists of 2 steps: first, a given amount of surfactant is extracted from a biologic fluid, and then it is fluorescently labelled to measure its accumulation at the air/liquid interface [74]. SAT can provide relatively quick results and is not biased by any intrinsic sample dilution: in fact, samples are tested at the same phosphatidylcholine concentration, and thus surfactant adsorption is measured in a fixed amount. SAT is more simple than complex techniques that fully describe surfactant biophysical activity but are not suitable to be used in clinical care [75]. SAT has been performed on amniotic fluid specimens from preterm neonates and found to be accurate to predict CPAP failure due to surfactant need (AUC: 0.84 [95\% CI: 0.824-0.856]) [76]. Moreover, surfactant adsorption significantly correlates with both LBC and LUS [76]. This confirms that surfactant efficiency, in terms of adsorption at air/liquid interface, is related to the available amount of surfactant and, finally, to lung aeration. As described above, lung aeration is influenced by several factors: therefore SAT, being one of these factors, may describe it more comprehensively than quantitative tests. This correlation between ultrasound-assessed lung aeration and surfactant adsorption has been found also in infants with neonatal ARDS induced by meconium aspiration [77]. Thus, interestingly, SAT could describe the role of surfactant efficiency also in neonatal ARDS and not only when surfactant is primarily lacking [78]. SAT needs 30-60 min and fluorometric readings, but this time can be shortened as we advance on the development pathway.

SMT is an old and simple assay based on the fact that surfactant-containing fluid, when vortexed, forms stable microbubbles of at least $<15 \mu \mathrm{m}$ [79]. Alike LBC, SMT has been used on gastric aspirate as well as amniotic and BAL fluid specimens to predict RDS and is quite diffused in some countries [80]. One study recently investigated it to predict CPAP failure due to surfactant need and found satisfactory reliability (AUC: 0.8 [95\% CI: 0.788-0.812]) [80]. There is a lack of physiopathology studies relating SMT results to lung aeration or other surfactant function measures, but SMT is the quickest functional test (only requiring few minutes). However, SMT needs a microscope and may be affected by a certain subjectivity influencing intra- and inter-observer variability [80]. Moreover, it has an intrinsic dilution problem because the drops of amniotic or gastric fluid may contain different amounts of surfactant, and thus the efficiency to create a stable bubble is not normalized for the available surfactant pool. 
Sensitivity

Estimate $(95 \% \mathrm{Cl}) \mathrm{TP} /(\mathrm{TP}+\mathrm{FN})$

$\begin{array}{ll}\text { LUS } & 0.836(0.768,0.886) 127 / 152 \\ \text { Chest X-rays } & 0.818(0.604,0.930) 18 / 22 \\ \text { LBC } & 0.700(0.543,0.821) 28 / 40 \\ \text { SAT } & 0.944(0.693,0.992) 17 / 18 \\ \text { SMT } & 0.714(0.524,0.850) 20 / 28\end{array}$

a

Specificity

Estimate $(95 \% \mathrm{Cl}) \mathrm{TN} /(\mathrm{FP}+\mathrm{TN})$

$\begin{array}{ll}\text { LUS } & 0.926(0.885,0.953) 224 / 242 \\ \text { Chest X-rays } & 0.882(0.725,0.955) 30 / 34 \\ \text { LBC } & 0.671(0.564,0.762) 57 / 85 \\ \text { SAT } & 0.696(0.485,0.847) 16 / 23 \\ \text { SMT } & 0.750(0.595,0.860) 30 / 40\end{array}$

b

AUC

Estimate $(95 \% \mathrm{Cl})$

LUS $\quad 0.952(0.951,0.953)$

Chest X-rays $\quad 0.800(0.792,0.808)$

LBC $\quad 0.703(0.696,0.710)$

SAT $\quad 0.840(0.824,0.856)$

SMT $\quad 0.800(0.788,0.812)$

c
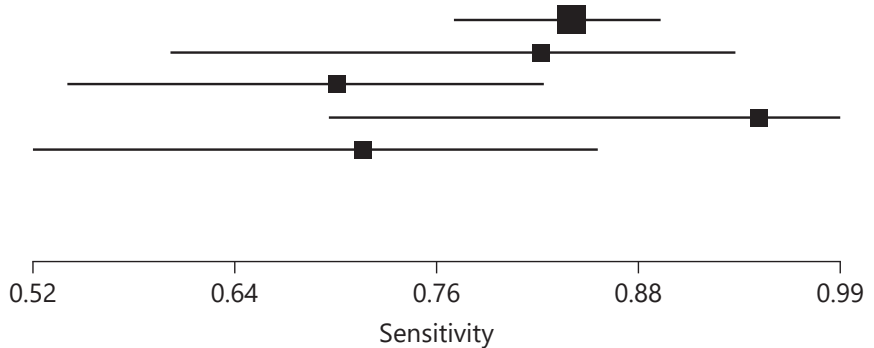

Sensitivity
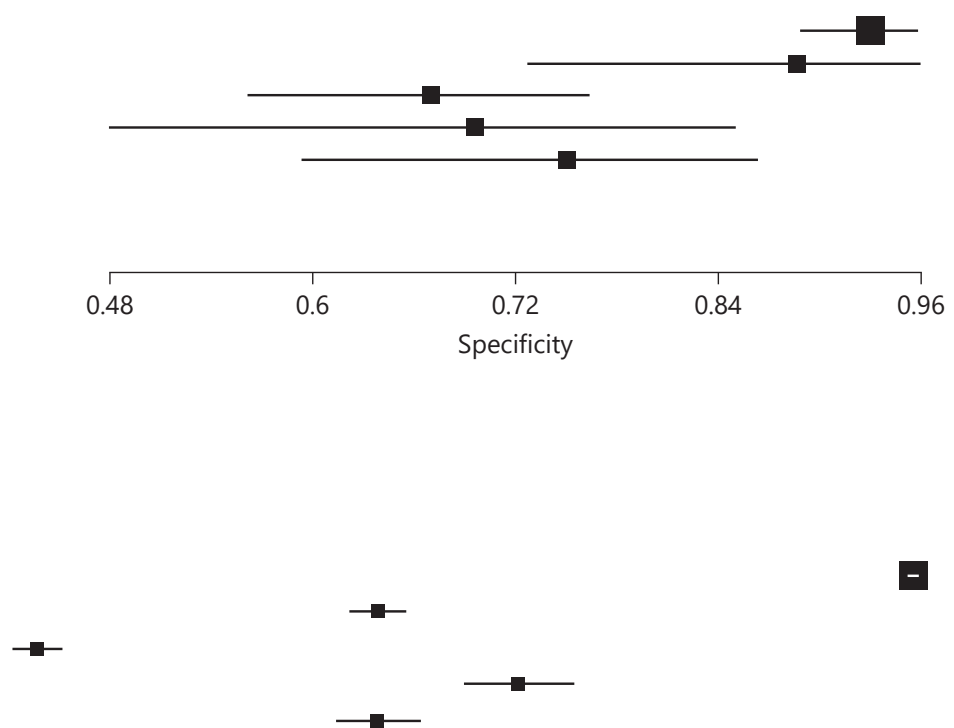

0.7

0.75

0.8

0.85

0.9

0.95
Fig. 3. Diagnostic accuracy of tools to personalize RDS management in preterm neonates. a-c Sensitivity, specificity, and AUC, respectively. LUS data are those derived from the meta-analyses of all diagnostic accuracy studies [37-42] on this technique (see Fig. 2). Data on chest X-rays, LBC, SAT, and SMT are extracted from Perri et al. [39], Raschetti et al. [66], Autilio et al. [76], and Bhatia et al. [80], respectively. Squares are proportional to the weight of populations used to study each technique (LUS: $n=443$; chest Xrays: $n=56$; LBC: $n=125$; SAT: $n=56$; and SMT: $n=68$ ). As a comparison, an $\mathrm{FiO}_{2}$ threshold of 0.30 has an AUC of approximately 0.8 calculated on a population of 297 neonates and sensitivity and specificity of approximately 80 and $60 \%$, respectively [14]. LUS data are referring to a cutoff value between 6 and 8. Horizontal lines represent 95\% CI. RDS, respiratory distress syndrome; LUS, quantitative lung ultrasound score; LBC, lamellar body count; SAT, surfactant adsorption test; SMT, stable microbubble test. 


\section{Conclusions}

The ideal tool to detect CPAP failure and manage RDS has some basic characteristics, such as:

- High accuracy and reproducibility

- Solid physiopathology background

- Quickly available results

- Relatively low cost

- Availability at the bedside

- Minimal invasiveness

- Easy to use

Many of the tools reviewed above met these characteristics. Advanced modified oxygenation metrics are physiologically sound and more informative than $\mathrm{FiO}_{2}$, yet they need to be investigated in specific studies clarifying their reliability to predict CPAP failure. LUS is ready to be widely implemented in clinical practice. EIT needs further industrial development and specific diagnostic accuracy studies. Surfactant biological tests have been subjected to specific diagnostic accuracy studies, but these have been conducted with single-centre design in small populations and may need further translational investigations and/or industrial development [81].

Figure 3 summarizes the diagnostic accuracy available for these tools in relation to the studied population sizes. The use of $0.30-\mathrm{FiO}_{2}$ threshold is associated with AUC ranging between 0.81 and 0.83 on a population of 297 neonates [14]: its sensitivity (approximately 80\%) was lower than that of LUS and SAT, and its specificity (ranging between 50 and 70\%) was lower than that of LUS or chest X-rays. Other tests have comparable results but large confidence intervals because they have been tested on smaller populations.

We advocate for a more accurate characterization of respiratory failure and a personalized approach for the management of RDS in preterm neonates. Lung ultrasound is immediately available and represents the commonest tool beyond oxygenation measurements: it is easily accessible as virtually every NICU has an ultrasound device; it may be used for various purposes beyond the prediction of CPAP failure and takes advantage of the large experience accumulated in adult critical care. However, the other candidate tools might be integrated in the NICU monitoring systems or represent easier point-ofcare diagnostics: their development and introduction on the clinical market should follow specific evidence-based guidelines [82]. We need specific involvement from the pharmaceutical and medical device industry to develop this field and finally make precision medicine available to all preterm neonates with RDS.

Personalization of RDS Management Is Possible

\section{Acknowledgements}

The authors are grateful to Alejandro Alonso Eugenio (Department of Biochemistry and Molecular Biology, Complutense University [Madrid, Spain]) for the artwork.

\section{Conflict of Interest Statement}

A/Prof. De Luca has received research and educational grants from Chiesi Pharmaceuticals spa and ABBVIE Inc. He served as a lecturer for Airway Therapeutics, Chiesi Pharmaceuticals spa, and ABBVIE Inc. Finally, he has been a member of advisory boards for Chiesi Pharmaceuticals spa and ABBVIE Inc. Dr. Shankar-Aguilera received a travel grant from Chiesi Pharmaceuticals spa. Prof. Carnielli received research grants and assistance and/or served as an advisory board member for Chiesi Pharmaceuticals spa. These companies produce surfactants or surfactant proteins, but had no role in design, preparation, review, approval of the manuscript, or decision to submit it for publication. The declared conflicts are all unrelated to the present manuscript. The other authors have no conflicts of interest to disclose.

\section{Funding Sources}

A/Prof. Tingay is supported by a National Health and Medical Research Council Clinical Career Development Fellowship (Grant ID 1053889) and the Victorian Government Operational Infrastructure Support Program (Melbourne, Australia). The funder did not participate in the work.

\section{Author Contributions}

A/Prof. De Luca conceptualized the project, performed the analysis, and drafted the manuscript; Dr. Autilio contributed to the literature search, data acquisition, and interpretation; Dr. Pezza contributed to the literature search, data acquisition, and interpretation; Dr. Shankar-Aguilera contributed to the data acquisition and interpretation; Prof. Carnielli contributed to the literature search, data acquisition, and interpretation; A/Prof. Tingay contributed to the literature search, data acquisition, and interpretation and supervised the project. All authors critically revised the manuscript for important intellectual content and approved the final manuscript as submitted. All authors agreed to be accountable for all aspects of the work in ensuring that questions related to the accuracy or integrity of any part of the work are appropriately investigated and resolved. 


\section{References}

1 Schmolzer GM, Kumar M, Pichler G, Aziz K, O’Reilly M, Cheung PY. Non-invasive versus invasive respiratory support in preterm infants at birth: systematic review and metaanalysis. BMJ. 2013 Oct 17;347:f5980.

2 Polin RA, Carlo WA; American Academy of Pediatrics, Committee on Fetus and Newborn. Surfactant replacement therapy for preterm and term neonates with respiratory distress. Pediatrics. 2014;133(1):156-63.

3 Sweet DG, Carnielli V, Greisen G, Hallman M, Ozek E, Plavka R, et al. European consensus guidelines on the management of neonatal respiratory distress syndrome in preterm infants: 2013 update. Neonatology. 2013; 103(4):353-68.

4 Bahadue FL, Soll R. Early versus delayed selective surfactant treatment for neonatal respiratory distress syndrome. Cochrane Neonatal Group. Cochrane Database Syst Rev. 2012;11(11):CD001456.

5 Raschetti R, Yousef N, Vigo G, Marseglia G, Centorrino R, Ben-Ammar R, et al. Echography-guided surfactant therapy to improve timeliness of surfactant replacement: a quality improvement project. J Pediatr. 2019;212: 137-43.e1.

6 Gregory GA, Kitterman JA, Phibbs RH, Tooley WH, Hamilton WK. Treatment of the idiopathic respiratory-distress syndrome with continuous positive airway pressure. N Engl J Med. 1971;284(24):1333-40.

7 Fujiwara T, Maeta H, Chida S, Morita T, Watabe $\mathrm{Y}, \mathrm{Abe} \mathrm{T}$. Artificial surfactant therapy in hyaline-membrane disease. Lancet. 1980; 1(8159):55-9.

8 Roberts D, Brown J, Medley N, Dalziel SR. Antenatal corticosteroids for accelerating fetal lung maturation for women at risk of preterm birth. Cochrane Database Syst Rev. 2017;3(3):CD004454.

9 Essouri S, Baudin F, Mortamet G, Beck J, Jouvet P, Emeriaud G. Relationship between diaphragmatic electrical activity and esophageal pressure monitoring in children. Pediatr Crit Care Med. 2019;20(7):e319-25.

10 Sweet DG, Carnielli V, Greisen G, Hallman M, Ozek E, Te Pas A, et al. European consensus guidelines on the management of respiratory distress syndrome: 2019 update. Neonatology. 2019;115(4):432-50.

11 Available from: https://extranet.who.int/rhl/ topics/newborn-health/care-newborn-infant/who-recommendation-continuous-positive-airway-pressure-therapy-treatmentpreterm-newborns Accessed 2020 Dec 5.

12 AARC Clinical Practice Guideline. Application of continuous positive airway pressure to neonates via nasal prongs, nasopharyngeal tube, or nasal mask: 2004 revision \& update. Resp Care. 2004;49:1100-8.
13 Waitz M, Engel C, Schloesser R, Rochwalsky U, Meyer S, Larsen A, et al. Application of two different nasal CPAP levels for the treatment of respiratory distress syndrome in preterm infants-"The OPTTIMMAL-Trial"-optimizing PEEP to the IMMAture lungs: study protocol of a randomized controlled trial. Trials. 2020;21(1):822.

14 Dargaville PA, Aiyappan A, De Paoli AG, Dalton RG, Kuschel CA, Kamlin CO, et al. Continuous positive airway pressure failure in preterm infants: incidence, predictors and consequences. Neonatology. 2013;104(1):814.

15 Fuchs $\mathrm{H}$, Lindner W, Leiprecht A, Mendler MR, Hummler HD. Predictors of early nasal CPAP failure and effects of various intubation criteria on the rate of mechanical ventilation in preterm infants of $<29$ weeks gestational age. Arch Dis Child Fetal Neonatal Ed. 2011; 96(5):F343-7.

16 Bard H. Postnatal fetal and adult hemoglobin synthesis in early preterm newborn infants. J Clin Invest. 1973;52(8):1789-95.

17 Jourdain G, De Tersant M, Dell'Orto V, Conti G, De Luca D. Continuous positive airway pressure delivery during less invasive surfactant administration: a physiologic study. J Perinatol. 2018;38(3):271-7.

18 ARDS Definition Task Force; Ranieri VM, Rubenfeld GD, Thompson BT, Ferguson ND, Caldwell E, et al. Acute respiratory distress syndrome: the Berlin definition. JAMA. 2012; 307(23):2526.

19 De Luca D, Piastra M, Chidini G, Tissieres P, Calderini E, Essouri $\mathrm{S}$, et al. The use of the Berlin definition for acute respiratory distress syndrome during infancy and early childhood: multicenter evaluation and expert consensus. Intensive Care Med. 2013 Dec;39(12): 2083-91.

20 Khemani RG, Smith LS, Zimmerman JJ, Erickson S. Pediatric acute respiratory distress syndrome: definition, incidence, and epidemiology. Pediatr Crit Care Med. 2015;16: S23-40.

21 De Luca D, van Kaam AH, Tingay DG, Courtney SE, Danhaive O, Carnielli VP, et al. The Montreux definition of neonatal ARDS: biological and clinical background behind the description of a new entity. Lancet Respir Med. 2017;5(8):657-66.

22 De Jaegere AP, van der Lee JH, Canté C, van Kaam AH. Early prediction of nasal continuous positive airway pressure failure in preterm infants less than 30 weeks gestation: early prediction of CPAP failure in preterms. Acta Paediatr. 2012;101(4):374-9.

23 Tridente A, De Martino L, De Luca D. Porcine vs. bovine surfactant therapy for preterm neonates with RDS: systematic review with biological plausibility and pragmatic meta-analysis of respiratory outcomes. Respir Res. 2019;20(1):28.
24 Wallace BC, Schmid CH, Lau J, Trikalinos TA. Meta-analyst: software for meta-analysis of binary, continuous and diagnostic data. BMC Med Res Methodol. 2009;9(1):80.

25 Hall RT. Indications for surfactant therapy: the aAPO2 coming of age. Pediatrics. 1999; 103(2):e25.

26 Wandrup JH. Quantifying pulmonary oxygen transfer deficits in critically ill patients. Acta Anaesthesiol Scand Suppl. 1995;107:37-44.

27 Restrepo RD, Hirst KR, Wittnebel L, Wettstein R. AARC clinical practice guideline: transcutaneous monitoring of carbon dioxide and oxygen: 2012. Respir Care. 2012;57(11): 1955-62.

28 Khemani RG, Rubin S, Belani S, Leung D, Erickson S, Smith LS, et al. Pulse oximetry vs. $\mathrm{PaO} 2$ metrics in mechanically ventilated children: Berlin definition of ARDS and mortality risk. Intensive Care Med. 2015;41(1):94102.

29 Chen W, Janz DR, Shaver CM, Bernard GR, Bastarache JA, Ware LB. Clinical characteristics and outcomes are similar in ARDS diagnosed by oxygen saturation/ $\mathrm{FiO} 2$ ratio compared with $\mathrm{PaO} 2 / \mathrm{FiO} 2$ ratio. Chest. 2015, 148(6):1477-83.

30 Rodriguez-Fanjul J, Jordan I, Balaguer M, Batista-Muñoz A, Ramon M, Bobillo-Perez S. Early surfactant replacement guided by lung ultrasound in preterm newborns with RDS: the ULTRASURF randomised controlled trial. Eur J Pediatr. 2020;179(12):1913-20.

31 Muniraman HK, Song AY, Ramanathan R, Fletcher KL, Kibe R, Ding L, et al. Evaluation of oxygen saturation index compared with oxygenation index in neonates with hypoxemic respiratory failure. JAMA Netw Open. 2019;2(3):e191179.

32 Thandaveshwara D, Chandrashekar Reddy AH, Gopalakrishna MV, Doreswamy SM. Saturation oxygenation pressure index: a non-invasive bedside measure for severity of respiratory disease in neonates on CPAP. Eur J Pediatr. 2020 Nov 20:1-6.

33 Raimondi F, Yousef N, Migliaro F, Capasso L, De Luca D. Point-of-care lung ultrasound in neonatology: classification into descriptive and functional applications. Pediatr Res. 2018 Jul 20:1-8.

34 Raimondi F, Migliaro F, Sodano A, Umbaldo A, Romano A, Vallone G, et al. Can neonatal lung ultrasound monitor fluid clearance and predict the need of respiratory support? Crit Care. 2012;16(6):R220.

35 Raimondi F, Migliaro F, Sodano A, Ferrara T, Lama S, Vallone G, et al. Use of neonatal chest ultrasound to predict noninvasive ventilation failure. Pediatrics. 2014;134(4):e1089-94.

36 Rodríguez-Fanjul J, Balcells C, Aldecoa-Bilbao V, Moreno J, Iriondo M. Lung ultrasound as a predictor of mechanical ventilation in neonates older than 32 weeks. Neonatology. 2016;110(3):198-203. 
37 Brat R, Yousef N, Klifa R, Reynaud S, Shankar Aguilera S, De Luca D. Lung ultrasonography score to evaluate oxygenation and surfactant need in neonates treated with continuous positive airway pressure. JAMA Pediatr. 2015; 169(8):e151797.

38 De Martino L, Yousef N, Ben-Ammar R, Raimondi F, Shankar-Aguilera S, De Luca D, et al. Lung ultrasound score predicts surfactant need in extremely preterm neonates. Pediatrics. 2018;142(3):e20180463.

39 Perri A, Riccardi R, Iannotta R, Di Molfetta DV, Arena R, Vento G, et al. Lung ultrasonography score versus chest $\mathrm{X}$-ray score to predict surfactant administration in newborns with respiratory distress syndrome. Pediatr Pulmonol. 2018;53(9):1231-6.

40 Gregorio-Hernández R, Arriaga-Redondo M, Pérez-Pérez A, Ramos-Navarro C, SánchezLuna M. Lung ultrasound in preterm infants with respiratory distress: experience in a neonatal intensive care unit. Eur J Pediatr. 2020; 179(1):81-9.

41 Vardar G, Karadag N, Karatekin G. The role of lung ultrasound as an early diagnostic tool for need of surfactant therapy in preterm infants with respiratory distress syndrome. Am J Perinatol. 2020 Jul 16. Epub ahead of print.

42 Pang H, Zhang B, Shi J, Zang J, Qiu L. Diagnostic value of lung ultrasound in evaluating the severity of neonatal respiratory distress syndrome. Eur J Radiol. 2019;116:186-91.

43 Razak A, Faden M. Neonatal lung ultrasonography to evaluate need for surfactant or mechanical ventilation: a systematic review and meta-analysis. Arch Dis Child Fetal Neonatal Ed. 2020;105(2): 164-71.

44 Mongodi S, Santangelo E, De Luca D, Rovida S, Corradi F, Volpicelli G, et al. Quantitative lung ultrasound: time for a consensus? Chest. 2020;158(2):469-70.

45 De Luca D, Yousef N. Pharmaceutical expenditure is unchanged with ultrasound-guided surfactant administration. Am J Perinatol. 2020 Aug 20.

46 Enghard P, Rademacher S, Nee J, Hasper D, Engert U, Jörres A, et al. Simplified lung ultrasound protocol shows excellent prediction of extravascular lung water in ventilated intensive care patients. Crit Care. 2015;19(1):36.

47 Yousef N, Vigo G, Shankar-Aguilera S, De Luca D. Semiquantitative ultrasound assessment of lung aeration correlates with lung tissue inflammation. Ultrasound Med Biol. 2020;46(5):1258-62.

48 Chiumello D, Mongodi S, Algieri I, Vergani GL, Orlando A, Via G, et al. Assessment of lung aeration and recruitment by CT scan and ultrasound in acute respiratory distress syndrome patients. Crit Care Med. 2018;46(11): 1761-8.

49 Kanto WP, Kuhns LP, Borer RC, Roloff DW. Failure of serial chest radiographs to predict recovery from respiratory distress syndrome. Am J Obstet Gynecol. 1978;131(7):757-60.
50 De Jaegere AP, Deurloo EE, van Rijn RR, Offringa $M$, van Kaam $\mathrm{AH}$. Individualized lung recruitment during high-frequency ventilation in preterm infants is not associated with lung hyperinflation and air leaks. Eur J Pediatr. 2016;175(8):1085-90.

51 Escourrou G, De Luca D. Lung ultrasound decreased radiation exposure in preterm infants in a neonatal intensive care unit. Acta Paediatr. 2016;105(5):e237-9.

52 Gomond-LeGoff C, Vivalda L, Foligno S, Loi B, Yousef N, De Luca D. Effect of different probes and expertise on the interpretation reliability of point-of-care lung ultrasound. Chest. 2020;157(4):924-31.

53 Brusa G, Savoia M, Vergine M, Bon A, Copetti R, Cattarossi L. Neonatal lung sonography: interobserver agreement between physician interpreters with varying levels of experience. J Ultrasound Med. 2015;34(9):1549-54.

54 Mazmanyan P, Kerobyan V, Shankar-Aguilera S, Yousef N, De Luca D. Introduction of point-of-care neonatal lung ultrasound in a developing country. Eur J Pediatr. 2020; 179(7):1131-7.

55 Singh Y, Tissot C, Fraga MV, Yousef N, Cortes RG, Lopez J, et al. International evidencebased guidelines on point of care ultrasound (POCUS) for critically ill neonates and children issued by the POCUS Working Group of the European Society of Paediatric and Neonatal Intensive Care (ESPNIC). Crit Care. 2020;24(1):65.

56 Frerichs I, Amato MB, van Kaam AH, Tingay DG, Zhao Z, Grychtol B, et al. Chest electrical impedance tomography examination, data analysis, terminology, clinical use and recommendations: consensus statement of the TRanslational EIT developmeNt stuDy group. Thorax. 2017;72(1):83-93.

57 Frerichs I, Dargaville PA, van Genderingen H, Morel DR, Rimensberger PC. Lung volume recruitment after surfactant administration modifies spatial distribution of ventilation. Am J Respir Crit Care Med. 2006;174(7): 772-9.

58 Tingay DG, Pereira-Fantini PM, Oakley R, McCall KE, Perkins EJ, Miedema M, et al. Gradual aeration at birth is more lung protective than a sustained inflation in preterm lambs. Am J Respir Crit Care Med. 2019; 200(5):608-16.

59 Miedema M, de Jongh FH, Frerichs I, van Veenendaal MB, van Kaam AH. Changes in lung volume and ventilation during surfactant treatment in ventilated preterm infants. Am J Respir Crit Care Med. 2011;184(1):1005 .

60 Bhatia R, Davis PG, Tingay DG. Regional volume characteristics of the preterm infant receiving first intention continuous positive airway pressure. J Pediatr. 2017;187:80-8.e2.
61 Heiring C, Verder H, Schousboe P, Jessen TE, Bender L, Ebbesen F, et al. Predicting respiratory distress syndrome at birth using a fast test based on spectroscopy of gastric aspirates: 2 . Clinical part. Acta Paediatr. 2020;109(2):28590.

62 Dietl P, Haller T. Exocytosis of lung surfactant: from the secretory vesicle to the air-liquid interface. Annu Rev Physiol. 2005;67(1): 595-621.

63 Danhaive O, Chapin C, Horneman H, Cogo PE, Ballard PL. Surface film formation in vitro by infant and therapeutic surfactants: role of surfactant protein B. Pediatr Res. 2015;77(2): 340-6.

64 Neerhof MG, Dohnal JC, Ashwood ER, Lee IS, Anceschi MM. Lamellar body counts: a consensus on protocol. Obstet Gynecol. 2001; 97(2):318-20.

65 Besnard AE, Wirjosoekarto SA, Broeze KA, Opmeer BC, Mol BW. Lecithin/sphingomyelin ratio and lamellar body count for fetal lung maturity: a meta-analysis. Eur J Obstet Gynecol Reprod Biol. 2013;169(2):177-83.

66 Raschetti R, Centorrino R, Letamendia E Benachi A, Marfaing-Koka A, De Luca D. Estimation of early life endogenous surfactant pool and CPAP failure in preterm neonates with RDS. Respir Res. 2019;20(1):75.

67 Verder H, Ebbesen F, Fenger-Grøn J, Henriksen TB, Andreasson B, Bender L, et al. Early surfactant guided by lamellar body counts on gastric aspirate in very preterm infants. Neonatology. 2013;104(2):116-22.

68 Jobe A, Jacobs H, Ikegami M. Lack of correlation of severity of lung disease with the phosphatidylcholine concentration in fetal lung fluid from premature lambs at 133-136 days gestational age. J Dev Physiol. 1984;6(5):417-21.

69 Cavicchioli P, Zimmermann LJ, Cogo PE, Badon T, Giordano G, Torresin M, et al. Endogenous surfactant turnover in preterm infants with respiratory distress syndrome studied with stable isotope lipids. Am J Respir Crit Care Med. 2001;163(1):55-60.

70 Costa S, Zecca E, De Luca D, De Carolis MP, Romagnoli C. Efficacy of a single dose of antenatal corticosteroids on morbidity and mortality of preterm infants. Eur J Obstet Gynecol Reprod Biol. 2007;131(2):154-7.

71 Jobe AH, Ikegami M, Jacobs HC, Jones SJ. Surfactant pool sizes and severity of respiratory distress syndrome in prematurely delivered lambs. Am Rev Respir Dis. 1983;127(6): 751-5.

72 Gunasekara L, Schoel WM, Schürch S, Amrein MW. A comparative study of mechanisms of surfactant inhibition. Biochim Biophys Acta. 2008;1778(2):433-44.

73 De Luca D, Vázquez-Sánchez S, Minucci A, Echaide M, Piastra M, Conti G, et al. Effect of whole body hypothermia on inflammation and surfactant function in asphyxiated neonates. Eur Respir J. 2014;44(6):1708-10. 
74 Ravasio A, Cruz A, Pérez-Gil J, Haller T. High-throughput evaluation of pulmonary surfactant adsorption and surface film formation. J Lipid Res. 2008;49(11):2479-88.

75 De Luca D, Lopez-Rodriguez E, Minucci A, Vendittelli F, Gentile L, Stival E, et al. Clinical and biological role of secretory phospholipase A2 in acute respiratory distress syndrome infants. Crit Care. 2013;17(4):R163.

76 Autilio C, Echaide M, Benachi A, MarfaingKoka A, Capoluongo ED, Pérez-Gil J, et al. A noninvasive surfactant adsorption test predicting the need for surfactant therapy in preterm infants treated with continuous positive airway pressure. J Pediatr. 2017; 182:66-73.e1.
77 Autilio C, Echaide M, Shankar-Aguilera S, Bragado R, Amidani D, Salomone F, et al. Surfactant injury in the early phase of severe meconium aspiration syndrome. Am J Respir Cell Mol Biol. 2020;63(3):327-37.

78 De Luca D, Minucci A, Tripodi D, Piastra M, Pietrini D, Zuppi C, et al. Role of distinct phospholipases A2 and their modulators in meconium aspiration syndrome in human neonates. Intensive Care Med. 2011;37(7): 1158-65.

79 Pattle RE, Kratzing CC, Parkinson CE, Graves L, Robertson RD, Robards GJ, et al. Maturity of fetal lungs tested by production of stable microbubbles in amniotic fluid. Br J Obstet Gynaecol. 1979;86(8):615-22.
80 Bhatia R, Morley CJ, Argus B, Tingay DG, Donath S, Davis PG. The stable microbubble test for determining continuous positive airway pressure (CPAP) success in very preterm infants receiving nasal CPAP from birth. Neonatology. 2013;104(3):188-93.

81 De Luca D, Capoluongo E, Rigo V; Study Group on Secretory Phospholipase in Paediatrics (SSPP). Secretory phospholipase A2 pathway in various types of lung injury in neonates and infants: a multicentre translational study. BMC Pediatr. 2011;11:101.

82 Huddy JR, Ni M, Misra S, Mavroveli S, Barlow J, Hanna GB. Development of the point-ofcare key evidence tool (POCKET): a checklist for multi-dimensional evidence generation in point-of-care tests. Clin Chem Lab Med. 2019;57(6):845-55. 